\title{
Behavior of a Scale Factor for the Wiener Integral of a Stochastic Fourier Transform
}

\author{
Young Sik Kim \\ Department of Mathematics, Research Institute of Natural Sciences, Industry -University Cooperation Foundation, Hanyang \\ University, 222 Wangsimni-ro, Seongdong-gu, Seoul 04763, Korea
}

\begin{abstract}
We investigate the behavior of a sacle factor for the Wiener integral of a stochastic Fourier transform of a measure on the abstract Wiener space.
\end{abstract}

Keywords—abstract Wiener space; Wiener integral.; stochastic Fourier transform

\section{INTRODUCTION}

In [1] and [2], R. H. Cameron and W. T. Martin developed Wiener integration theory about transformations of Wiener integrals on the Wiener space.

In [3] R. H. Cameron and W. T. Martin investigated the behavior of measure and measurability under change of scale in Wiener space.

L.Gross[6] and J.Kuelb[14] and H.H. Kuo[15] developed thr Wiener integration theory on the abstract Wiener space .

In [7] and [9], Y.S.Kim proved relationships among the Wiener integral and the analytic Feynman integral.

In this paper, we investigate the behavior of a scale factor for the Wiener integral about the stochastic Fourier transform $\mathrm{F}(x)=\int_{H} \exp \left\{i(h, x)^{\sim}\right\} d \mu(h)$ of a measure $\mu \in M(H)$, where $M(H)$ is the class of complex valued countably additive measure $\mu \in M(H)$ defined on the Borel class of $\mathrm{H}$ on the abstract Wiener space.

\section{DEFINITIONS AND PRELIMINARYS}

Let $\mathrm{H}$ be a real separable infinite dimensional Hilbert space with inner product $\langle\because, \cdot\rangle$ and norm $|\cdot|=\sqrt{\langle\because,\rangle}$.

Let $\|\cdot\|_{0}$ be a measurable norm on $\mathrm{H}$ with respect to the Gauss measure $\mu \in M(H))$. Let B denote the completion of $\mathrm{H}$ with respect to $\|\cdot\|_{0}$. Let $\mathrm{i}$ denote the natural injection from $\mathrm{H}$ into $\mathrm{B}$. The adjoint operator $i^{*}$ of $\mathrm{i}$ is one-to-one and maps $B^{*}$ continuously onto a dense subset of $H^{*}$, where $H^{*}$ and $B^{*}$ are topological duals of $\mathrm{H}$ and $\mathrm{B}$, respectively. By identifying $\mathrm{H}$ with $H^{*}$ and $B^{*}$ with $i^{*} B^{*}$, we have a triplet $\left(B^{*}, \mathrm{H}, \mathrm{B}\right)$ such that $B^{*} \mathrm{C} H^{*} \equiv H \mathrm{C} B$ and $<\mathrm{h}, \mathrm{x}>$ $=(\mathrm{h}, \mathrm{x})$ for all $x$ in $B^{*}$ and $h$ in $\mathrm{H}$, where $(\because$,$) denotes the$ natural dual pairing between $B^{*}$ and $\mathrm{B}$. By a well known result of Gross [6], $\mu \cdot i^{-1}$ has a unique countably additive extension $\mathrm{m}$ to the Borel $\sigma$-algebra $B(\mathrm{~B})$ on $\mathrm{B}$. Then $(\mathrm{B}, \mathrm{H}, \mathrm{m})$ is called an abstract Wiener space and $\mathrm{m}$ is called a Wiener measure. We denote the Wiener integral of a functional $\mathrm{F}$ by $\int_{B} F(x) d m(x)$.
Let $\left\{e_{j}\right\}_{j=1}^{\infty}$ denote a complete orthonormal system in $\mathrm{H}$ such that $e_{j}^{\prime} \mathrm{s}$ are in $B^{*}$. For each $h \in H$ and $\mathrm{x} \in \mathrm{B}$, we define a stochastic inner product $(\because,)^{\sim}$ between $\mathrm{H}$ and $\mathrm{B}$ as follows :

$$
(h, x)^{\sim}=\left\{\begin{array}{cc}
\lim _{n \rightarrow \infty} \sum_{j=1}^{n}\left\langle h, e_{j}\right\rangle\left(e_{j}, x\right), & \text { if the limit exists } \\
0 & \text { otherwise } .
\end{array}\right.
$$

It is well known that for every $h \in H,(h, x)^{\sim}$ exists for $\mu-$ a.e. $\mathrm{x}$ in $\mathrm{B}$ and it has a Gaussian distribution with mean zero and variance $|h|^{2}$. Furthermore, it is easy to show that $(h, x)^{\sim}=(h, x)$ for $\mu-$ a. e. $\mathrm{x}$ in $\mathrm{B}$ if $\mathrm{h} \in B^{*},(h, x)^{\sim}$ is essentially independent of the complete orthonormal set used in its definition, and finally that if $\left\{\left(h_{1}, x\right)^{\sim}, \cdots,\left(h_{n}, x\right)^{\sim}\right\}$ is an orthonormal set of elements in $\mathrm{H}$, then $\left(h_{1}, x\right)^{\sim}, \cdots,\left(h_{n}, x\right)^{\sim}$ are independent Gaussian functionals with mean zero and variance one. Note that if both $\mathrm{h}$ and $\mathrm{x}$ are in $\mathrm{H}$, then $(h, x)^{\sim}=\langle h, x\rangle$.

Throughout this paper, let $R^{n}$ denote the $\mathrm{n}$-dimensional Euclidean space and let $\mathrm{C}, C_{+}, C_{+}^{\sim}$ denote the complex numbers, the complex numbers with positive real part, and the non-zero complex numbers with nonnegative real part, respectively.

Definition 2.1. Let $(\mathrm{B}, \mathrm{H}, \mathrm{m})$ be an abstract Wiener space.

Let $C_{+}=\{z \mid \operatorname{Re}(z)>0\}$ and $C_{+}^{\sim}=\{z \mid \operatorname{Re}(z) \geq 0\}$. Let $\mathrm{F}$ be a complex-valued scale invariant measurable function on $\mathrm{B}$ such that the integral

$$
J_{F}(r)=\int_{B} F\left(r^{-\frac{1}{2}} x\right) d m(x)
$$

exists for all real $\mathrm{r}>0$. If there exists an analytic function $J_{F}^{*}(z)$ analytic on $C_{+}$such that $J_{F}^{*}(r)=J_{F}(r)$ for all real $\mathrm{r}>$ 0 , then we define $J_{F}^{*}(z)$ to be the analytic Wiener integral of $\mathrm{F}$ over B with parameter $z \in C_{+}$and for each $z \in C_{+}$, we write

$$
\int_{B}^{a n w_{z}} F(x) d m(x)=J_{F}^{*}(z)
$$

Let $\mathrm{q}$ be a non-zero real number and let $\mathrm{F}$ be a function whose analytic Wiener integral exists for each $z \in C_{+}$. If the limit exists, then we call it the analytic Feynman integral of F over B with parameter q, and we write

$$
\int_{B}^{a n f_{q}} F(x) d m(x)=\lim _{z \rightarrow-i q} \int_{B}^{a n f_{q}} F(x) d m(x)
$$


where $\mathrm{z}$ approaches -iq through $C_{+}$and $i^{2}=-1$.

Now we introduce the Fresnel class of functions in the abstract Wiener space.

Definition 2.2. Let Let $(B, H, m)$ be an abstract Wiener space. The Fresnel class $\mathrm{F}(\mathrm{B})$ is defined by

$$
F(B)=\left\{[F]: F(x)=\int_{H} \exp \left\{i(h, x)^{\sim}\right\} d \mu(h), x \in B\right\} .
$$

where $\mu \in M(H)$ and $\mathrm{M}(\mathrm{H})$ is the space of complex valued countably additive measure $\mu$ defined on $\mathrm{B}(\mathrm{H})$, the Borel class of $\mathrm{H}$. We will identify a function with its s-equivalencd class and think of $F(B)$ as a collection of functions on $B$ rather than as a class of equivalence classes.

The following is a well-known Wiener integration formula for the Wiener integral on the abstract Wiener space.

Theorem 2.3. Let Let $(B, H, m)$ be an abstract Wiener space. and let $\mathrm{F}$ be a function on $\mathrm{B}$ of the form $F(x)=$ $f\left((h, x)^{\sim}\right)$, where $f: R \rightarrow \boldsymbol{C}$ is a Lebesgue measurable function. Then

$$
\int_{B} f\left((h, x)^{\sim}\right) d m(x)=\left(\frac{1}{2 \pi|h|^{2}}\right)^{\frac{1}{2}} \int_{R} f(u) \cdot \exp \left\{-\frac{1}{2|h|^{2}} u^{2}\right\} \mathrm{du}
$$

where " = " means that if either side exists, then both sides exists and they are equal.

Remark. In the next section, we will several times the following formula :

$$
\int_{R} \exp \left\{-a u^{2}+i b u\right\} d u=\sqrt{\frac{\pi}{a}} \exp \left\{-\frac{1}{2|h|^{2}} u^{2}\right\}
$$

where a is a complex number with $\operatorname{Re}(\mathrm{a})>0$, and $\mathrm{b}$ is a real number and $i^{2}=-1$.

\section{THE MAIN RESULT}

First, we obtain the Wiener integral of the stochastic Fourier transform of a measure $\mu \in M(H)$ in the Fresnel class $\mathrm{F}(\mathrm{B})$ on the abstract Wiener space.

Theorem 3.1 Let $(\mathrm{B}, \mathrm{H}, \mathrm{m})$ be an abstract Wiener space. Let $\mathrm{F}$ be the stochastic Fourier transform of a measure $\mu \in$ $M(H)$ in the Fresnel class $\mathrm{F}(\mathrm{B})$ of the form (2.5). Then for real $\rho>0$, the Wiener integral of the function $F$ exists and is of the form :

$$
\int_{B} F(\rho x) d m(x)=\int_{H} \exp \left\{-\frac{\rho^{2}}{2}|h|^{2}\right\} \mathrm{du},
$$

Proof. By the Wiener integration formula in Theorem 2.3, we can easily have that for real $\rho>0$,

$$
\begin{gathered}
\int_{B} F(\rho x) d m(x) \\
=\int_{B}\left[\int_{H} \exp \left\{i(h, \rho x)^{\sim}\right\} d \mu(h)\right] d m(x)
\end{gathered}
$$

$$
\begin{gathered}
=\int_{H}\left[\int_{B} \exp \left\{i(h, \rho x)^{\sim}\right\} d m(x)\right] d \mu(h) \\
=\int_{H} \exp \left\{-\frac{\rho^{2}}{2}|h|^{2}\right\} d \mu(h) .
\end{gathered}
$$

Note that for all real $\rho>0, \int_{B} F(\rho x) d m(x) \leq\|\mu\|<\infty$. Therefore, the Wiener integral exists for all real $\rho>0$.

By the above result, we can investigate a very interesting behavior of the scale factor for the Wiener integral which was first defined by the author in [13].

Definition 3.2. We define the scale factor for the Wiener integral by the real number $\rho>0$ of the absolute value of the Wiener integral :

$$
\mathrm{G}(\rho)=\left|\int_{B} F(\rho x) d m(x)\right|
$$

where $\mathrm{G}: \mathrm{R} \rightarrow \mathrm{R}$ is a real valued function on $\mathrm{R}$.

Remark. For $\mathrm{x} \in \mathrm{B}$, we shall interprete it as followings :

(1). For real $\rho>1, \rho \mathrm{x}$ is a magnification of $\mathrm{x} \in \mathrm{B}$.

(2). For real $0<\rho<1, \rho \mathrm{x}$ is a minimization of $\mathrm{x} \in \mathrm{B}$.

(a). Behavior of a scale factor for the Wiener integral in the Fresnel class $F(B)$ on the abstract Wiener space.

Whenever we magnify and minimize $x \in B$, the Wiener integral varies very interestingly according to the varying scale factor :
(1) $\int_{B} F\left(\frac{1}{100} x\right) d m(x)=\int_{H} \exp \left\{-\frac{1}{2 \times 10^{4}}|h|^{2}\right\} d \mu(h)$
(2) $\int_{B} F\left(\frac{1}{10} x\right) d m(x)=\int_{H} \exp \left\{-\frac{1}{2 \times 10^{2}}|h|^{2}\right\} d \mu(h)$
(3) $\int_{B} F(x) d m(x)=\int_{H} \exp \left\{-|h|^{2}\right\} d \mu(h)$
(4) $\int_{B} F(10 x) d m(x)=\int_{H} \exp \left\{-\frac{10^{2}}{2}|h|^{2}\right\} d \mu(h)$
(5) $\int_{B} F(100 x) d m(x)=\int_{H} \exp \left\{-\frac{10^{4}}{2}|h|^{2}\right\} d \mu(h)$.

(b). Interpretation of a scale factor for the Wiener integral in the Fresnel class $F(B)$ on the abstract Wiener space.

(1) Whenever the scale factor $\rho>1$ is increasing, the Wiener integral decreases very rapidly.

(2) Whenever the scale factor $0<\rho<1$ is decreasing, the Wiener integral increases very rapidly.

(3) The scale factor $\rho>0$ plays a very interesting behavior of the magnification and the minimization of the Wiener integral !

(4) The function $\mathrm{G}(\rho)=\left|\int_{B} F(\rho x) d m(x)\right|$ is a decreasing function of a scale factor $\rho>0$, whenever $\rho \rightarrow \infty$ : 


$$
\begin{aligned}
& \text { (a). } 0 \leq\left|\int_{B} F(\rho x) d m(x)\right| \leq\|\mu\| \\
& \text { (b). } \lim _{\rho \rightarrow 0}\left|\int_{B} F(\rho x) d m(x)\right|=\|\mu\| \\
& \text { (c). } \lim _{\rho \rightarrow \infty}\left|\int_{B} F(\rho x) d m(x)\right|=0
\end{aligned}
$$

(5). Whenever the scale factor $\rho>0$ increases, the Wiener integral decreases very rapidly. Whenever the scale factor $\rho>$ 0 decreases, the Wiener integral increases very rapidly !

Finally, we introduce the Motivation and the Application of the Wiener integral :

\section{Remark.}

\section{(1) Motivation : The solution of the heat equation}

$$
\frac{\partial U}{\partial t}=-H U, \quad U(0, \cdot)=\varphi(\cdot)
$$

is

$$
\begin{gathered}
U(t, \varepsilon)=\left(e^{-t H} \varphi\right)(\varepsilon) \\
=E\left[e^{-\int_{0}^{t} V(x(s)+\varepsilon) d s} \cdot \varphi(x(t)+\varepsilon)\right],
\end{gathered}
$$

where $\varphi \in L_{2}\left[R^{d}\right]$ and $\varepsilon \in R^{d}$ and $\mathrm{x}(\cdot)$ is a $R^{d}$ - valued continuous function defined on $[0, \mathrm{t}]$ such that $\mathrm{x}(0)=0$ and $\mathrm{E}$ denotes the expectation with respect to the Wiener path starting at time $\mathrm{t}=0$ and $H=-\Delta+\mathrm{V}$ is the energy operator(or, Hamiltonian) and $\Delta$ is a Laplacian and $\mathrm{V}: R^{d} \rightarrow R$ is a potential. This formula (4.12) is called the Feynman-Kac formula.

(2). Application of the Feynman-Kac formula(in various settings) have been given in the area : diffusion equation, the spectral theory of the schrodin̈ger operator, quantum mechanics, statistical physics.(For more details, see the book [8].

\section{ACKNOWLEDGMENT}

THANKS 1. I am very gratitude to professors : R.H. Cameron and M.T.Martin and D.A. Storvick in [1] [5] who give me a very nice research area about the structure of the change of scale and the change of scale formula and about the every harmonious relationships among the Wiener integral and the analytic Wiener integral and the analytic Feynman integral.

Those papers give me an excellent motivation about the new concept of the scale factor for the Wiener integral.

THANKS 2. I am very gratitude to professors, G.W. Johnson and M.L.Lapidus to research about the Feynman integral theory from their book in [8].
THANKS 3. I am very gratitude to the National Research Foundation of the Republic of Korea supporting the research fund for my work.

Research fund of this paper is supported by NRF2017R1A6A3A11030607.

\section{REFERENCES}

[1] R. H. Cameron and W. T. Martin, On transformations of Wiener integrals under translations. Ann. of Math. 45. (1944), 386 - 396.

[2] R. H. Cameron and W. T. Martin, Transformations for Wiener integrals under a general class of linear transformations. Trans. Amer. Math. Soc. 58. No 2, (1945), 184 - 219.

[3] R. H. Cameron and W. T. Martin, The behavior of measure and measurability under change of scale in Wiener space. Bull. Amer. Math. Soc. 53, (1947) 130-137.

[4] R.H.Cameron and D.A.Storvick, Relationships between the Wiener Integral and the Analytic Feynman Integral. Supplemento ai Rendiconti del Circolo Matematico di Palermo, Serie II-numero. 17. (1987), 117133.

[5] R.H.Cameron and D.A.Storvick, Change of scale Formulas for Wiener Integral. Supplemento ai Rendiconti del Circolo Matematico di Palermo, Serie II-numero. 17. (1987), 105-115.

[6] L.Gross, Abstract Wiener Space, Proc.Fifth Berkeley Symposium Math. Stat. Prob. (1965), 31 - 42

[7] Y. S. Kim, A change of scale formula for Wiener Integrals of cylinder functions on the abstract Wiener space. Internat. J. of Math. and Math.Sci. 21. No 1 (1998), 73-78.

[8] G.W. Johnson and M.L. Lapidus, The Feynman integral and Feynman's Operational Calculus, Oxford Science Publications. (2000).

[9] Y. S. Kim, A change of scale formula for Wiener Integrals of cylinder functions on abstract Wiener space II. Internat.J.of Math.and Math.Sci. 25. No 4, (2001), 231-237.

[10] Y.S.Kim, The behavior of the first variation under the Fourier Feynman transform on abstract Wiener spaces. Journal of Fourier Analysis and Application. 12, No 3, (2006), 233-242.

[11] Y.S.Kim,. Fourier Feynman Transform and analytic Feynman integrals and convolutions of a Fourier transform of a measure on Wiener spaces. Houston Journal of Mathematics. 36, No 4 (2010), 1139 - 1158.

[12] Y.S.Kim, The behavior of the first variation of Fourier transforms of a measure on the Fourier-Feynman transform and convolution. Numerical Functional Analysis and Optimization.. 31, (2016), 699-718.

[13] Y. S. Kim, Behavior of a scale factor for Wiener integrals and a Fourier Stieltjes transform on the Wiener space. Applied Mathematics. No 9, (2018), 488-495.

[14] J.Kuelbs. Abstract Wiener Spaces and Applications to Analysis, Pacific J. Math. 31, (1969), 433-450.

[15] H.H.Kuo, Gaussian Measures in Banach Spaces, Lecture Notes in Math. 463. Springer-Verlag, Berlin. (1975). 